\title{
Capturing Transient Molecular Structures on the Millisecond Time Scale for EM Imaging
}

\author{
Fa-Qing Zhao,* and Roger Craig*
}

*Dept. of Cell Biology, University of Massachusetts Medical School, Worcester, MA 01655

Imaging intermediates of biological processes is a key step in understanding the structural basis of biological function. Because intermediates are commonly short-lived, lasting only milliseconds, the main methods used to capture them have been conventional imaging of analog states with extended lifetimes, or rapid freezing of intermediates, following brief ( $\sim$ msecs) exposure to conditions that initiate the structural change, induced either by spraying or by uncaging of a caged precursor, with subsequent observation by cryo-EM.[1,2]

We have developed a simpler solution to this problem that captures structural intermediates on the millisecond time scale for subsequent imaging by conventional techniques, such as negative staining. The procedure consists of the following steps: 1. the specimen (macromolecular assembly such as filament, tubule or virus) is applied to a carbon-coated grid; 2. the grid is flushed briefly with an "activating" solution that induces a structural change; 3 . immediately (e.g. $10 \mathrm{msec}$ ) following, the grid is rapidly flushed with uranyl acetate, which we show below acts as an ultrarapid fixative; 4. the stain is allowed to dry as with conventional negative staining. Steps 2 and 3 are accomplished by drawing into a $1 \mathrm{ml}$ pipette tip first $520 \mu \mathrm{l}$ uranyl acetate, then $60 \mu \mathrm{l}$ air, and finally $40 \mu \mathrm{l}$ activating solution (the air gap prevents mixing of the solutions). The contents are then rapidly expelled from the pipette over the grid. The activating solution contacts the grid first, but remains only transiently (e.g. 10 or $30 \mathrm{msec}$, measured electronically) before being flushed away with the stain.

To successfully capture structural changes occurring on the millisecond time scale, not only must the activating solution be rapidly replaced by the stain, but the stain must rapidly fix the transiently induced structure. The key finding that validates our approach is our demonstration that uranyl acetate, and to a lesser extent tannic acid, fix protein molecular structure in as little as $10 \mathrm{msec}$. This is clear from our observation that exposure of actin and myosin filaments to uranyl acetate for 10 msec is sufficient to fully preserve them against changes that normally induce rapid and major alterations in their molecular structure. An example is shown in Fig. 1. Native scallop myosin filaments in relaxing medium (MgATP, low $\mathrm{Ca}^{2+}$ ) are in the switched "off" state (low myosin ATPase, interaction with actin filaments inhibited) and exhibit helically ordered crossbridges (Fig. 1a). When such filaments are exposed to $\mathrm{Ca}^{2+}$, they are switched "on" (myosin ATPase is activated, actin binding becomes possible). Structurally the myosin heads start to disorder within $10 \mathrm{msec}$ and are fully disordered within $30 \mathrm{msec}$, extending away from the filament backbone (Fig. 1b).[3] We find that if the relaxed filaments are exposed instead to uranyl acetate for only $10 \mathrm{msec}$ before being activated, they retain their helical order (Fig. 1c; Table 1). Thus uranyl acetate preserves their molecular structure in as little as $10 \mathrm{msec}$. Similar results are obtained with tannic acid (Fig. 1d). Fixation appears to preserve both ionic and hydrophobic bonds. This is suggested by the finding (Table 1) that uranyl acetate also acts to preserve: 1. myosin filament integrity against treatment with high salt (which otherwise dissolves myosin filaments); 2 . actin-myosin binding against treatment with ATP (which otherwise rapidly dissociates myosin from actin); $3 . \mathrm{Mg}^{2+}$-induced actin paracrystals against dissociation in low $\left[\mathrm{Mg}^{2+}\right]$; and 4. actin filaments against forming paracrystals in high $\left[\mathrm{Mg}^{2+}\right]$. Tannic acid acts similarly although generally more slowly than uranyl acetate. 
We conclude that this approach, involving rapid staining following rapid activation, should be of general utility for studying transient molecular changes in many systems (e.g. motor protein function, assembly-disassembly processes etc.). It should also be possible to combine this simple method of rapid fixation with other imaging methods such as cryo-EM and rotary shadowing.[4]

\section{References}

[1] J. Berriman and N. Unwin (1994). Ultramicroscopy 56:241-252.

[2] J.F. Ménétret et al. (1991). J. Mol. Biol. 219:139-144.

[3] F.Q. Zhao and R. Craig (2002). Biophys. J. 82:6a.

[4] This work was supported by grants from the NIH (AR34711, HL62468).

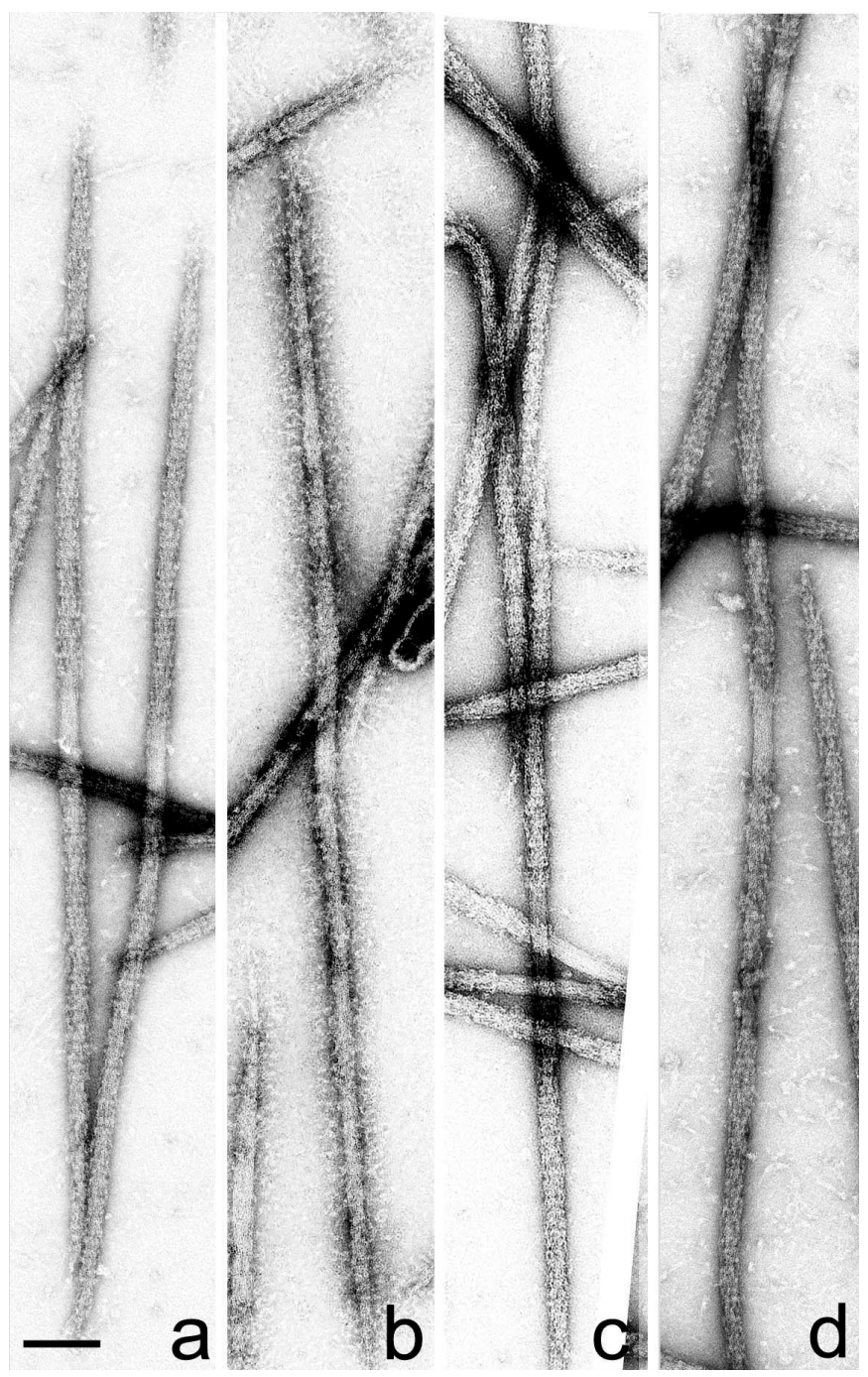

Figure 1. Negatively stained (1\% UA) myosin filaments. (a) relaxed (low $\mathrm{Ca}^{2+}$ ) - heads helically ordered; (b) activated (high $\mathrm{Ca}^{2+}$ ) - disordered; (c) as in (b), but with prior exposure $(10 \mathrm{msec})$ to $1 \% \mathrm{UA}$ ordered; (d) as in (b), but with prior exposure (10 $\mathrm{msec}$ ) to $0.25 \%$ tannic acid - ordered. Bar $=100 \mathrm{~nm}$.
Table 1. Fast fixation properties of uranyl acetate and tannic acid.

\begin{tabular}{lccccc}
\hline & $\begin{array}{c}\text { Thick } \\
\text { filaments }\end{array}$ & $\begin{array}{c}\text { Acto- } \\
\text { S1 }\end{array}$ & $\begin{array}{c}\text { Actin } \\
\text { PCs }\end{array}$ & $\begin{array}{c}\text { Actin } \\
\text { filaments }\end{array}$ \\
\hline Treatment & $+\mathrm{Ca}^{2+}$ & + salt & $+\mathrm{ATP}$ & $-\mathrm{Mg}^{2+}$ & $+\mathrm{Mg}^{2+}$ \\
\hline \multicolumn{6}{c}{$1 \% \mathrm{UA}$} \\
\hline $10 \mathrm{sec}$ & $\mathrm{Y}$ & $\mathrm{Y}$ & $\mathrm{Y}$ & $\mathrm{Y}$ & $\mathrm{Y}$ \\
$30 \mathrm{msec}$ & $\mathrm{Y}$ & $\mathrm{Y}$ & $\mathrm{Y}$ & $\mathrm{Y}$ & $\mathrm{Y}$ \\
$10 \mathrm{msec}$ & $\mathrm{Y}$ & $\mathrm{Y}$ & $\mathrm{Y}$ & $\mathrm{Y}$ & $\mathrm{Y}$ \\
\hline \multicolumn{7}{c}{$0.25 \%$} & Tannic acid \\
\hline $10 \mathrm{sec}$ & $\mathrm{Y}$ & $\mathrm{Y}$ & $\mathrm{Y} / \mathrm{N}^{*}$ & $\mathrm{Y} / \mathrm{N}^{* *}$ & $\mathrm{Y}$ \\
$30 \mathrm{msec}$ & $\mathrm{Y}$ & $\mathrm{Y}$ & $\mathrm{N}$ & $\mathrm{N}$ & $\mathrm{Y}$ \\
$10 \mathrm{msec}$ & $\mathrm{Y}$ & $\mathrm{Y}$ & $\mathrm{N}$ & $\mathrm{N}$ & $\mathrm{N}$ \\
\hline
\end{tabular}

Table shows protective effect of uranyl acetate and tannic acid against disordering of thick filaments by $\mathrm{Ca}^{2+}$ or high salt $(0.6 \mathrm{M}$ $\mathrm{NaCl}$ ), dissociation of myosin heads (S1) from actin by $\mathrm{MgATP}$, dissociation of actin paracrystals by low $\mathrm{Mg}^{2+}$, and formation of actin paracrystals by high $\mathrm{Mg}^{2+}$. Grids were flushed with uranyl acetate or tannic acid for $10 \mathrm{msec}, 30 \mathrm{msec}$, or $\sim 10 \mathrm{sec}$ before being exposed to activating solution, and subsequently negatively stained with $1 \%$ UA. $\mathrm{Y}$, fixes; $\mathrm{N}$, doesn't fix; * requires $1 \mathrm{~min}$ fixation; $* *$ requires 3 min fixation. 